\title{
Vivências e estágios no SUS: ênfase na atenção primária em saúde
}

\section{Andressa Carine Kretschmer}

Universidade Federal de Santa Maria | Brasil

dessa felicia@hotmail.com

\section{Resumo}

0 presente trabalho narra as experiências de acadêmicos na condução do estágio e vivência no Sistema Único de Saúde (SUS), denominado VER-SUS na 15a Coordenadoria de Saúde do Rio Grande do Sul, programa de extensão com o intuito de aproximar o graduando da realidade do Sistema Único de Saúde. Para concretização desta proposta fez-se o uso de rodas de conversas com profissionais de saúde, visitas a unidades de saúde, contato com movimentos sociais e com o movimento estudantil. A presente imersão obteve a duração de 15 dias, tendo ocorrido em 15 de fevereiro a 1 de março de 2013. Após o término da vivência, foi unânime a constatação de a que participação no programa foi um divisor de águas no que tange a carreira acadêmica e convicções. Ela despertou em muitos dos participantes o anseio de trabalhar nos meandros do sistema ou mesmo buscar pela especialização na área contemplada.

\section{Palavras-chave}

Educação em Saúde; Sistema Único de Saúde; Estágios. 


\section{Introdução}

O Sistema Único de Saúde (SUS) é um dos maiores sistemas públicos de saúde existente no mundo. Foi criado em 1988 pela Constituição Federal Brasileira e regulamentado pelas Leis n.o 8080/1990 e n⿳0 8.142/1990. Sua abrangência inclui desde procedimentos simples, atenção básica de saúde, até transplantes de órgãos, (BRASIL, 1988, 1990a, 1990b). O SUS é de acesso integral, universal e gratuito para toda a população. Conforme o previsto pela Constituição os convênios privados atuam como complemento ao sistema público de saúde. (BRASIL, 1988, 1990a, 1990b).

Para Campos, (2007) o SUS ainda é uma reforma social incompleta, uma política de construção recente, um sistema que necessita melhorar seu prestígio e superar as barreiras que ainda limitam seu fortalecimento, para assim tornar-se um sistema de saúde mais eficaz e com vasta capilaridade. Para isto não somente é necessária a ampliação de recursos, mas a constituição de um forte movimento social e em defesa do bem-estar e da construção de políticas públicas de proteção social, assim como o empoderamento da população quanto ao funcionamento das organizações públicas.

Muitas críticas são direcionadas ao SUS, principalmente no que se refere à sua gestão, atuação e limitações na sua capacidade de assistir toda sua clientela. Porém, para que haja mudanças nesta perspectiva, é necessária a capacitação dos profissionais antes de sua chegada ao sistema, trabalhando-se assim com os futuros atores sanitários e sociais durante a sua trajetória na academia. (BRASIL, 2006). Uma das ferramentas adotadas com a finalidade de capacitar e aproximar o graduando do SUS são as Vivências e Estágios no Sistema Único de Saúde-VER-SUS, que tem como finalidade aproximar o graduando da realidade prática do modelo de saúde pública, sensibilizar e humanizar os futuros atores para que assim em um futuro próximo sejam comprometidos eticamente com as diretrizes do SUS, (BRASIL, 2012).

O VER-SUS ocorre no Brasil desde 2002, trata-se de uma atividade de Educação Permanente em Saúde que propicia aos estudantes de cursos de graduação e áreas afins experimentar os espaços de aprendizagem no SUS, o cotidiano de trabalho nas organizações redes e sistemas. É um dispositivo educativo com vistas à formação dos trabalhadores em saúde, voltado para o SUS, com o compromisso ético-político para as necessidades de saúde e sociais da população. 0 mesmo é realizado tradicionalmente nas férias universitárias das instituições de ensino superior. Seu gerenciamento e agendamento são realizados por meio de acadêmicos organizados em executivas e profissionais que ansiarem em realizá-lo em sua localidade que 
Vivências e estágios no SUS: ênfase na atenção primária em saúde

assim se disponibilizarem a construir o escopo, cujo custeio das atividades é adotado por entidades do estado ou em nível federal, (BRASIL, 2012).

Dentro do programa VER-SUS/BRASIL o acadêmico passa por uma imersão no cotidiano de trabalho do SUS na atenção, gestão, na relação com o controle social, movimentos sociais e escola formadora. $\mathrm{O}$ escopo abrange estudantes, professores, comunidade e serviços de saúde, e o acadêmico passa por visitas nas unidades de saúde, rodas de debates, místicas e dinâmicas diversas, (LIRA et al, 2013).

Por tratar-se de uma política de governo, o VER-SUS foi abandonado em algumas localidades por um período de tempo. Em agosto de 2011 o projeto VER-SUS/BRASIL foi retomado pela Escola de Saúde Pública/RS, com apoio do Ministério da Saúde em parceria com a Rede Unida, Rede Colaborativa de Governo, Universidade Federal do Rio Grande do Sul (UFRGS) e União Nacional dos Estudantes (UNE), e é executado até os dias atuais, (FERLA, RAMOS, LEAL, CARVALHO, 2013).

Os estágios de vivência não são recentes no Brasil, nem mesmo no campo da saúde, a história de sua realização é antiga e descende de outras experiências já realizadas. Esses estágios inicialmente foram idealizados pelo movimento estudantil que criticava a forma de ensino das universidades e apostando na maneira de ensino próxima da atuação profissional, (FERLA, RAMOS, LEAL, CARVALHO, 2013).

O VER-SUS surge como uma ferramenta a fim de questionar a universidade que deveria direcionar seu ensino, pesquisa e extensão na formação de profissionais comprometidos com as necessidades da sociedade e não em apenas repetir "ideias" já construídas, mas sim, refleti-las, propô-las, transforma-las a fim socializar conhecimentos na construção de uma sociedade mais igualitária, (ROSA, 2006).

A realização de estágios vivência deu-se a partir do final dos anos 1980 nos assentamentos rurais brasileiros, sendo realizado pelos estudantes das Ciências Rurais. Inicialmente esta proposta foi organizada pelas executivas dos cursos de Agronomia e de Medicina Veterinária. Com o passar dos anos o Estágio Interdisciplinar de Vivência (EIV) passou a inserir acadêmicos das mais diversas áreas. A proposta surgiu da necessidade de se entender de maneira crítica o modelo agrário adotado no Brasil. Pode-se citar também como agente de impulsão para o VER-SUS a realização em 2001 da Escola de Verão no estado do Rio Grande do Sul, na qual participaram cerca de 50 estudantes do curso de Medicina das instituições de ensino do Rio Grande do Sul. E em 2002 ocorre a realização das Vivências e Estágios na Realidade do SUS-VER-SUS, com apoio da Escola de Saúde Pública do Rio Grande do Sul (ESP-RS) e em parceria com o Ministério da Saúde, (FERLA, RAMOS, LEAL, CARVALHO, 2013). 
Vivências e estágios no SUS: ênfase na atenção primária em saúde

A realização das vivências-estágios surge, como o exposto acima, do movimento estudantil, que se preocupava com os estudantes da área da saúde por não conhecerem o funcionamento do SUS, sua gestão, suas atividades peculiares, seus objetivos e sua abrangência. Cabe salientar que muitas destas preocupações e o sentimento de despreparo dos graduandos é consequência da insuficiência das atividades práticas, muitas vezes com reduzida carga horária, e de grades curriculares que não contemplam de maneira satisfatória o ensino de saúde pública, (MENDES, 2012).

A vivência realizada em Palmeira das Missões-RS foi organizada pelos acadêmicos que compõem o Coletivo Social de Mudanças na Saúde (Cosmus) juntamente com integrantes do Diretório Central do Estudante (DCE) da Universidade Federal de Santa Maria (UFSM), que se disponibilizaram a construir o escopo, juntamente com o cronograma de atividades, dinâmicas e visitas a unidades de saúde. 0 projeto construído pela comissão organizadora obteve aprovação do orçamento, e do cronograma, sendo financiado pela Escola de Saúde Pública do Rio Grande do Sul (ESP/RS), que custeou as despesas dos estudantes com alimentação, transporte, e materiais didáticos.

0 presente relato tem como objetivo destacar as experiências dos acadêmicos participantes das Vivências e Estágios na realidade do Sistema Único de Saúde da 15a Coordenadoria de Saúde na cidade polo de Palmeira das Missões, Rio Grande do Sul, e sua interface para a promoção de mudanças sanitárias e sociais.

\section{Método}

O presente estudo utiliza a narrativa como forma de apresentar e problematizar a experiência vivida por estudantes universitários na atuação e condução de projeto de extensão no campo da saúde. 0 estágio ocorreu nos meses de férias acadêmicas, de 15 de fevereiro a 1 de março de 2013, em cidades da 15ª Coordenadoria Regional de Saúde do estado do Rio Grande do Sul (Palmeira das Missões, São Pedro das Missões, Redentora e Ronda Alta), tendo como base o município de Palmeira das Missões. Contou com a participação de acadêmicos provenientes de Instituições de ensino superior público e privados do estado do Rio Grande do Sul; Universidade Federal de Santa Maria (UFSM), Universidade de Passo Fundo (UPF), Pontifícia Universidade Católica (PUC) e Universidade Federal do Rio Grande do Sul (UFRGS), dos mais diversos cursos: administração, economia, enfermagem, nutrição, fisioterapia, psicologia, teatro, odontologia, e comunicação social.

A seleção dos estudantes que iriam participar deste estágio foi realizada de modo on-line, através do site do Observatório de Tecnologias de Informação e Comunicação em Sistemas e 
Serviços de Saúde-OTICSi conveniado ao Ministério da Saúde, via envio de cartas de intenções. A OTICS é uma parceria do Instituto de Comunicação e Informação Científica e Tecnológica em Saúde (ICICT), da Fiocruz, com a UFRGS para pesquisar, desenvolver e disponibilizar soluções metodológicas e tecnológicas que contribuam para o fortalecimento do SUS, por meio de um conjunto de soluções em informática, (OTICS, 2015).

Dentre os critérios de seleção para a participação do projeto, destacaram-se: a interdisciplinaridade (inclusão de cursos de graduações diferentes), diferentes semestres/fases de graduação, interinstitucionalidade, paridade de gênero, raça e sexualidade, a fim de assegurar a diversidade, a quebra de pré-conceitos e o trabalho em equipe multidisciplinar, assim como o que ocorre no dia-a-dia dos trabalhadores do SUS. Foram classificados para o projeto 20 estagiários e quatro facilitadores cuja tarefa era auxiliar o aprendizado durante a vivência, sendo os mesmos; um do curso de Ciências Econômicas e outro da Enfermagem da UFSM, Centro de Educação Superior Norte, instituição que sediou o evento. Os outros dois; um do curso de Psicologia da PUC-RS e o outro do curso de Teatro da UFRGS, cada facilitador teve a incumbência de conduzir as atividades de um grupo de cinco graduandos.

Priorizou-se pela multidisciplinaridade, embora a maior parte dos graduandos inscritos correspondesse ao curso de enfermagem. Pode-se desfrutar durante a vivência além das tradicionais conversas com gestores, profissionais e visitas em unidades de saúde, o contato com os movimentos sociais; tais como o Movimento dos Trabalhadores Sem Terra (MST), o Movimento dos Pequenos Agricultores (MPA), e o movimento estudantil. Houve também no decorrer do projeto a visita a um assentamento sem-terra e a uma reserva indígena, pois se entendeu que o estágio não deveria ficar restrito apenas a debates em torno das visitas a unidades de saúde, mas sim a compreender também outros processos de luta pertinentes.

Após as visitas às unidades de saúde e localidades exploradas, houve a realização de debates com profissionais da saúde, dinâmicas, místicas, relatórios e sistematizações conduzidas pelos facilitadores. Também foram utilizados, como subsídio para a formação, textos, palestras, e informações trazidas por ex-participantes do VER-SUS.

\section{Resultados e discussões}

Durante os dias iniciais do projeto, no município base, realizaram-se discussões em torno das lutas da sociedade para a consolidação do SUS, o papel do movimento estudantil como propulsor deste processo, e as fragilidades e pontos fortes do SUS na saúde pública. Estas foram conduzidas pelos facilitadores, comissão de apoio, ex-participantes do programa e pósgraduandos. 
Vivências e estágios no SUS: ênfase na atenção primária em saúde

Pode-se compreender a realidade do funcionamento de algumas unidades de saúde dos municípios explorados (Palmeira das Missões, São Pedro das Missões, Redentora e Ronda Alta), do qual se visitou algumas das Estratégias de Saúde da Família (ESF) de todos os munícipios. Percebeu-se que municípios de menor porte possuem uma capilaridade menor das políticas em ação, necessitando assim de uma boa conexão com a coordenadoria de saúde para que o acesso aos demais serviços de sejam garantidos.

Nos municípios de Palmeira das Missões e Ronda Alta pode-se explorar a realidade do SUS em nível de atenção terciária (um hospital com demandas regionais e outro que supre demandas regionais e com especialidades no âmbito da saúde mental respectivamente), este foi o considerado o nível de atuação do SUS que conforme os relatos dos estudantes mais carecia de mudanças, no sentido de que o sistema necessita agilizar o atendimento dos usuários. Também cabe salientar que a região da coordenadoria explorada não possui hospitais cujo atendimento é $100 \%$ SUS.

Em Redentora, cidade que concentra em seu território parte de uma reserva indígena, explorou-se a conversa com os profissionais de uma unidade básica de saúde a respeito do acesso dos remanescentes nativos ao SUS. A distância entre a reserva e os municípios revelou-se como um empecilho para o acesso a atenção terciária.

Na cidade base do projeto visitou-se: o ambulatório de saúde mental, este que mediante a constituição de todos os profissionais necessários tornar-se-ia um Centro de Atenção Psicossocial I (CAPS I), o Centro de Referência Especializada em Saúde do Trabalhador (CEREST), o Centro de Referência de Assistência Social (CRAS) e a 15 a Coordenadoria Regional de Saúde (CRS). Uma das questões levantadas pelo grupo tratou-se de inexistência do Núcleo de Apoio à Saúde da Família (NASF), principalmente pelo fato de se tratar da cidade polo da 15a CRS, assim como a incompletude da política do CAPS I.

$\mathrm{Na}$ base, também explorou por debates em torno dos processos de luta da sociedade, através do contato com o MST, MPA e seus respectivos representantes locais, outro processo de luta abordado foi o movimento estudantil sendo este conduzido por acadêmicos pertencentes ao Diretório Acadêmico, Diretório Central dos Estudantes, e Coletivos Estudantis. Também em Palmeira das Missões realizou-se a participação a um Conselho Municipal de Saúde e a visita um assentamento desencadeado pelo MST. Posteriormente a todas as visitas realizadas realizou-se a discussão das políticas públicas em vigência, os pontos fortes, fragilidades e proposições para melhorias no sistema.

Muitos participantes desta edição do VER-SUS possuíam uma visão negativa do sistema púbico de saúde, vislumbravam o SUS apenas como um sistema precário, falido e caracterizado pelo excesso de filas. Além disso, alguns estudantes desconheciam ou tinham uma visão limitada 
Vivências e estágios no SUS: ênfase na atenção primária em saúde

de seu funcionamento. Com o decorrer do estágio VER-SUS, muitos passaram a afirmar que a existência do SUS é fundamental. Consideram sua base teórica perfeita, porém na prática as coisas não ocorrem de maneira satisfatória, pois o SUS não consegue chegar até algumas localidades e atender toda sua clientela, é um sistema que necessita de profissionais capacitados e humanizados para atuar e cumprir com suas demandas. As principais alternativas levantadas para mudar a realidade existente foram; a humanização dos profissionais e dos futuros agentes sanitários e sociais através da educação permanente em saúde e o questionamento da universidade quanto às atuais grades curriculares existentes e o seu papel social. Conforme Ceccim, (2004), o cotidiano do trabalho e da universidade deve ser tido como lugar aberto à revisão permanente e gerar o desconforto com os lugares "como estão/como são". Sendo o questionamento a abertura para a prática da Educação Permanente em Saúde.

Os serviços de saúde visitados demonstraram-se abertos à discussão e à apresentação dos profissionais que atuavam em suas unidades. Notou-se que havia localidades nas quais o SUS funcionava muito bem, havia uma boa gestão, havia o trabalho multidisciplinar, e empenho dos profissionais, críticas e sugestões foram bastante valorizadas pelos profissionais das localidades visitadas.

A visita e o acampamento na reserva indígena foram de fundamental importância, considerando as dificuldades que os remanescentes de etnias nativas possuem de acessar o sistema; dificuldades de saneamento básico e o pré-conceito existente aos remanescentes por parte dos graduandos, muitos dos estudantes pensavam que os mesmos se encontrariam seminus e praticando a agricultura rudimentar, porém o índio atual possui grande parte dos hábitos encontrados nas cidades.

Um dos grandes objetivos do VER-SUS é estimular os graduandos a fazer parte do movimento estudantil; integrar os Diretórios Acadêmicos de seus cursos, DCE de suas universidades, integrar coletivos estudantis e compor executivas de cursos em âmbitos regional e estadual, para que assim organizados possam repensar as grades curriculares de seus cursos e o papel social da universidade e a lutar por mudanças sociais no que tange a desigualdades e injustiças.

A presença de estudantes de diversos cursos de graduação, inclusive além das ciências da saúde, possibilitou com que os saberes em torno das políticas públicas fossem vistos com abrangência, situações tais como a do controle social do SUS, da qual todos como cidadãos têm a oportunidade de participar e acompanhar as ações de saúde, bem como a aplicação dos recursos financeiros.

Houve durante a vivência a participação de uma reunião do Conselho Municipal de Saúde, assim como posteriormente debateu-se a respeito da importância da participação e do 
Vivências e estágios no SUS: ênfase na atenção primária em saúde

conhecimento da população quanto ao funcionamento do controle social do SUS. A assistência em saúde é direito de todos e dever do estado, e para seu funcionamento adequado é necessário que os usuários conheçam os instrumentos de representação institucional e as medidas cabíveis. A forma mais direta trata da participação nos Conselhos Municipais de Saúde, onde a sociedade é representada por seus segmentos sendo $50 \%$ da representação colegiada.

Para Oliveira, Ianni \& Dallari, (2013) a participação dos usuários do sistema nos conselhos de saúde trata-se de um sentimento de pertencimento do cidadão a sociedade, porém ainda é necessário a disposição de mais informações à população em geral para que assim haja a quebra da corporação nas decisões, situação da qual ocorre em algumas localidades.

Durante as vivências houve a desinibição do grupo para questionamentos, posicionamentos e opiniões. A importância da observação e anotação daquilo que foi apresentado, para assim compor o relato do dia, foi uma proposição metodológica constantemente enfatizada. Quanto às dificuldades, destacam-se: cansaço para a realização das atividades propostas; dificuldades logísticas; divulgação insuficiente da vivência; incompreensão do site da OTICS; desconhecimento dos modelos de financiamento por parte da comissão organizadora (Cosmus e DCE); gastos exorbitantes por parte do estado na contratação da empresa terceirizada a prestar os serviços de alimentação e transporte durante a vivência e dificuldades no cumprimento de horários propostos pelo cronograma, gerando a necessidade de atividades alternativas ao programa inicial.

Pode se compreender que o Sistema Único de Saúde é uma obra construída pelas pessoas, um sistema do qual foi fruto de muitas lutas e de movimentos no sentido do qual a saúde é um direito social, criado pelo povo e para cuidar da população, embora muitas pessoas não o reconheçam como tal.

Mesmo o VER-SUS não sendo a única prática que incorpora questões em torno do eixo SUS, a vivência foi uma alternativa viável para contribuir com o processo de formação, pois a mesma aproxima o acadêmico com a realidade da saúde da região abordada, para que profissionais formados possam compreendê-lo e, assim, colaborar para seu desenvolvimento. A formação na área da saúde é antes de tudo um instrumento para as transformações e aperfeiçoamentos do SUS.

Já dizia Paulo Freire, (2006, p. 47), do processo de ensino-aprendizagem, "ensinar não é transferir conhecimento, mas sim criar possibilidades de sua construção", o que vai de encontro ao ambiente de trabalho do SUS, do qual é de suma importância que se o obtenha desde o ingresso na graduação, uma vez que é o contato direto com o usuário. Corrobora Ceccim (2005) ao tratar de que a educação em saúde faz-se próximo a realidade existencial, sendo esta 
Vivências e estágios no SUS: ênfase na atenção primária em saúde

realizada não somente na atenção em saúde, mas sim no compromisso de manter a exequibilidade dos princípios sanitários da política e gestão do SUS.

\section{Conclusões}

Para os acadêmicos participantes foi unânime a constatação de que a participação no VER-SUS foi um divisor de águas na carreira acadêmica, devido ao incentivo à interdisciplinaridade, partindo-se do ponto de vista de que a saúde deve ser vista de maneira integral e não em "caixas". Deu àqueles que teriam suas trajetórias acadêmicas afastadas do ensino de saúde pública a possibilidade de conhecer a realidade do SUS, principalmente ao nível da atenção básica, conhecendo a atuação conjunta das equipes multidisciplinares e o funcionamento das unidades básicas de algumas localidades.

Para a maior parte dos graduandos participantes do estágio VER-SUS, o Sistema único de Saúde não é mais visto apenas como um sistema de baixa qualidade, mas sim como um sistema que necessita de humanização e atenção por parte dos gestores e profissionais, e que na prática o seu funcionamento tem muito a melhorar.

Como aspectos a serem observados para as próximas vivências, sugere-se uma melhor distribuição do tempo para que assim evite-se o cansaço e o desgaste, uma divulgação mais eficaz do projeto, agregando estudantes de universidades mais distantes. Muitos alunos relataram o interesse em participar do programa devido à universidade não contemplar de maneira satisfatória o ensino de saúde pública, com frequência ausente de seus respectivos programas político pedagógicos de graduação. Por fim, pode-se dizer que amadureceu em alguns e despertou em outros o anseio de posteriormente destinar seus estudos para o tema do SUS, ou mesmo fazê-lo futuramente seu ambiente de trabalho.

\section{Referências}

BRASIL. Constituição Federal de 1988. Diário Oficial da União, 1988.

BRASIL. Presidência da República. Lei no 8.080, de 19 de Setembro. Ministério da Saúde, Brasília, DF, 1990a.

BRASIL. Presidência da República. Lei no 8.142, de 28 de Dezembro. Ministério da Saúde, Brasília, DF, 1990b.

BRASIL. Ministério da Saúde. Política Nacional de Educação Permanente em Saúde. Ministério da Saúde, Brasília/DF, 2006. 
Vivências e estágios no SUS: ênfase na atenção primária em saúde

BRASIL. Ministério da Saúde. Vivências e estágios na realidade do sistema único de saúde. Ministério da Saúde, Brasília/DF, 2012.

CAMPOS, G.W.S. Reforma política e sanitária: a sustentabilidade do SUS em questão? Ciência: Saúde Coletiva, Rio de Janeiro, v. 12, n. 2, p. 301-306, 2007.

CECCIM, R. B. Educação Permanente em Saúde: descentralização e disseminação de capacidade pedagógica na saúde. Ciência: Saúde Coletiva. v. 10, n. 4, p. 975-986, 2005.

CECCIM, R. B. Educação Permanente em Saúde: desafio ambicioso e necessário. Interface Comunic, Saúde, Educação, vol. 9, n. 16, p. 161-177, 2004.

FERLA, A. A; RAMOS, A. S; LEAL, M. B; CARVALHO, M.S. Caderno de Textos do VER-SUS/ Brasil. Porto Alegre: Rede Unida, 2013.

FREIRE, P. Pedagogia da Autonomia: saberes necessários à prática educativa. São Paulo. Paz e Terra; 2006.

LIRA, J. C. G et al. VER-SUS: Um Relato de Experiência sobre uma Vivência-Estágio na Realidade do Sistema Único de Saúde. Revista de Enfermagem UFPE On Line, v. 7, p. 1-7, 2013.

MENDES, F. M. S et al. VER-SUS: relato de vivências na formação de Psicologia. Psicologia: Ciência e Profissão. Brasília, v. 32, n. 1, p. 175-186, 2012.

OBSERVATÓRIO de Tecnologias em Informação e Comunicação em Sistemas e Serviços de Saúde. Otics. Disponível em <http://www.otics.org/estacoes-de-observacao/versus $>$. Acesso em: 22 out. 2015.

OLIVEIRA, A.M.C; IANNI, A.M.Z; DALLARI, S.G. Controle social no SUS: discurso, ação e reação. Ciência: saúde coletiva, Rio de Janeiro, v. 18, n. 8, p. 2329-2338, 2013.

ROSA, I. M et al. Movimento Estudantil Propósitos, Extensão Universitária e Educação Popular: contribuições para o debate. Revista Brasileira de Extensão Universitária, v. 4, n. 2, p. 26-31, 2006.

i Ver: <http://versus.otics.org>. 
Vivências e estágios no SUS: ênfase na atenção primária em saúde

Experiences and stages in public health: emphasis on primary health care

\section{ABSTRACT}

This paper chronicles the academic experiences in conducting of an extension project in the Sistema Único de Saúde (SUS - Brazilian Public Health System), called VER-SUS, outreach program with the aim of learn about reality of the Brazilian Public Health System, which the academy often meets an inefficient critical vision of the Brazilian public health. To achieve this proposal, it was made by using conversations with health professionals, health centers visits, social movements and contact with the student activism. This experience occurs in 15 days, from February 15 to March 1, 2013. In the end of this experiences, it was unanimous in finding that the program was a watershed with respect to academic career and convictions. The stage awakened in many of the participants the desire to work in the system intricacies or even seek the expertise in the covered area.

\section{Keywords}

Health Education; Public Health in Brazil; Internship Experience.

Original submetido em: 20 set. 2015

Aceito para publicação em: 8 dez. 2015
Experiencias y pasantías en el Sistema Único de Saúde (SUS): énfasis en atención primaria de salud

\section{RESUMEN}

Este trabajo narra las experiencias académicas en la realización de una actividad de extensión en el Sistema Único de Saúde (SUS - sistema brasileño de Salud Pública) en la 15 Coordenadoria de Saúde do Rio Grande do Sul, llamado VER-SUS, programa de extensión que acerca a los estudiantes universitarios de la realidad del sistema de salud pública de Brasil. Para lograr esta propuesta se hizo conversaciones con profesionales de la salud, visitas a centros de salud, movimientos sociales y el contacto con el movimiento estudiantil. La experiencia duró 15 días, ocurrió el 15 febrero 1 marzo 2013. Al final del trabajo se concluyó de forma unánime que el programa fue un hito en lo que respecta a las enseñanzas académicas $\mathrm{y}$ profesionales. La pasantía despertó en muchos de los participantes el deseo de trabajar en los entresijos del sistema o incluso buscar la especialización en la misma área.

\section{Palabras clave}

Educación para la salud; Sistema de salud pública; Pasantía.

Sobre os autores:

\section{Andressa Carine Kretschmer}

Bacharel em Nutrição pela Universidade Federal de Santa Maria (UFSM), Campus Palmeira das Missões. 